\title{
Transduction Sites of Vagal Mechanoreceptors in the Guinea Pig Esophagus
}

\author{
Vladimir P. Zagorodnyuk and Simon J. H. Brookes \\ Department of Human Physiology and Centre for Neuroscience, Flinders University of South Australia, \\ Adelaide, South Australia 5001
}

\begin{abstract}
Extrinsic afferent neurons play an essential role in both sensation and reflex control of visceral organs, but their specialized morphological peripheral endings have never been functionally identified. Extracellular recordings were made from fine nerve trunks running between the vagus nerve and esophagus of the guinea pig. Mechanoreceptors, which responded to esophageal distension, fired spontaneously, had low thresholds to circumferential stretch, and were slowly adapting. Calibrated von Frey hairs $(0.12$ $\mathrm{mN}$ ) were used to probe the serosal surface at 100-200 sites, which were mapped on a video image of the live preparation. Each stretch-sensitive unit had one to three highly localized receptive fields ("hot spots"), which were marked with Indian ink applied on the tip of the von Frey hair. Recorded nerve trunks were then filled anterogradely, using biotinamide in an artificial intracellular solution. Receptive fields were consistently associated with intraganglionic laminar endings (IGLEs) in myenteric
\end{abstract}

ganglia, but not with other filled neuronal structures. The average distance of receptive fields to IGLEs was $73 \pm 14 \mu \mathrm{m}$ (24 receptive fields, from 12 units; $n=5$ ), compared to $374 \pm 17 \mu \mathrm{m}$ for 240 randomly generated sites $(n=5 ; p<0.001)$. After maintained probing on a single receptive field, spontaneous discharge of units was inhibited, as were responses to distension. During adapted discharge to maintained distension, interspike intervals were distributed in a narrow range. This indicates that multiple receptive fields interact to encode mechanical distortion in a graded manner. IGLEs are specialized transduction sites of mechanosensitive vagal afferent neurons in the guinea pig esophagus.

Key words: vagus nerve; primary afferent; mechanoreceptor; intraganglionic laminar ending; guinea pig; esophagus; enteric nervous system
Visceral afferents to the gut are involved in many pathological conditions such as gastroesophageal reflux (which causes the symptoms of heartburn), gastroparesis, and the "functional disorders" such as irritable bowel syndrome, which have major medical and economic impact on the community (Talley et al., 1995). Sensations such as satiety, nausea, and pain are also mediated by these neurons. However they are also important in reflex control of normal gut functions, such as swallowing, gastric accommodation, and control of gastric acid secretion (Cervero, 1994; Sengupta and Gebhart, 1994). The efferent side of many of these reflexes is well understood, being mediated via parasympathetic pathways, which run in the vagus nerve, or via sympathetic neurons running in mesenteric nerves to the gut. Vagal efferent neurons in turn activate enteric neurons that comprise the final output to effector cells and sympathetic fibers modulate synaptic activity in the enteric nervous system (Furness and Costa, 1987). However, the details of the afferent side of these pathways are rather less clear.

Neuroanatomical techniques have characterized the morphology of extrinsic afferent nerve endings in the wall of the gastrointestinal tract, particularly from the vagus nerve (Clerc and Condamin, 1987; Berthoud and Powley, 1992; Berthoud et al., 1997; Phillips et al., 1997). Vagal afferent neurons give rise to three types of specialized endings in the gut wall. In myenteric ganglia, intraganglionic laminar endings (IGLEs) (Lawrentjew, 1929; Rodrigo et al., 1975) are found on the surfaces of myenteric ganglia. In the muscle layers, intramuscular arrays (IMAs) of afferent nerve fibers have been described (Berthoud et al., 1997; Phillips et al., 1997), and

Received March 13, 2000; revised May 22, 2000; accepted May 30, 2000.

This study was funded by AstraZeneca, Mölndal, Sweden, and S.J.H.B. was supported by the National Health and Medical Research Council of Australia. We thank Grant Hennig for his generous help with the mapping of video images and Bill Blessing, Marcello Costa, Ian Gibbins, Phil Jobling, and Judy Morris for commenting on this manuscript.

Correspondence should be addressed to Simon Brookes, Department of Human Physiology, Flinders University, GPO Box 2100, Adelaide, South Australia 5001. E-mail: simon.brookes@flinders.edu.au.

Copyright (C) 2000 Society for Neuroscience $0270-6474 / 00 / 206249-07 \$ 15.00 / 0$ there are also vagal afferent nerve endings in the mucosa (Berthoud and Patterson, 1996). There are fewer studies on the terminations of spinal afferents to the gastrointestinal tract, but these appear generally to give rise to nonspecialized free nerve endings (Cervero, 1994) in the mucosa, muscle, and serosa. Electrophysiologically, extrinsic mechanoreceptors to the gut have been extensively studied using extracellular recording techniques (Iggo, 1955; Leek, 1977; Sengupta and Gebhart, 1994; Page and Blackshaw, 1998). Among the vagal afferent fibers, mechanosensitive and chemosensitive mucosal receptors have been distinguished. Mechanoreceptors have been classified by their threshold, dynamic range, and rate of adaptation (Sengupta and Gebhart, 1994). However, it has not been possible, to date, to relate morphological and physiological studies of visceral afferent neurons and thus identify the endings of particular types of afferents. In this study, vagal mechanoreceptors to the guinea pig esophagus were studied electrophysiologically, and their morphology was subsequently determined by anterograde labeling from the recorded nerve trunk. Intraganglionic laminar endings were shown to be the transduction sites of vagal mechanoreceptors in the guinea pig esophagus. This novel combination of techniques now makes it possible to characterize the morphological features of functionally identified visceral afferent neurons.

\section{MATERIALS AND METHODS}

Close extracellular single unit recording. Guinea pigs $(n=57)$ were killed humanely by stunning and exsanguination, in a manner approved by the Animal Welfare Committee of Flinders University. The distal esophagus was opened up into a flat sheet. The mucosa was then removed, and the preparation was attached to a microprocessor-controlled tissue stretcher (Brookes et al., 1999). A fine vagal nerve trunk was dissected free of connective tissue and passed under a glass coverslip partition into a small chamber ( $1000 \mu \mathrm{l}$ volume) made from Sylgard (Dow Corning, Midland, MI) and filled with paraffin. Single-unit recordings were made differentially from the nerve trunk and from a strand of connective tissue, via platinum electrodes, on a MacLab 8s at 20,000 samples/sec, using Chart 3.6 software (ADI, Sydney, Australia). Single units were discriminated by amplitude and duration using Spike Histogram software (AD Instruments). Preparations were stretched by the tissue stretcher at 10-5000 $\mu \mathrm{m} / \mathrm{sec}$ and held for 3-60 sec, while monitoring circumferential tension. A 
a

Figure 1. Typical response of esophageal vagal afferents to circumferential stretch. $a$, Rapid stretch $(2 \mathrm{~mm}$ at $5 \mathrm{~mm} / \mathrm{sec})$ evoked an increase in firing above the spontaneous firing rate. After the removal of stretch, spontaneous firing was inhibited for $\sim 20$ sec. $b$, During slow stretch $(3 \mathrm{~mm}$ at $200 \mu \mathrm{m} / \mathrm{sec}$ ) there was a graded, approximately linear increase in firing rate. $c$, Combined data from 13 units show graded responses to rapid stretch $(5 \mathrm{~mm} / \mathrm{sec})$ of different amplitudes ( $1 \mathrm{~mm}$, filled circles; $2 \mathrm{~mm}$, open squares; and $3 \mathrm{~mm}$, filled triangles), with a graded poststimulus inhibition of firing. $d$, Combined data from 11 units respond to $2 \mathrm{~mm}$ of stretch at $5 \mathrm{~mm} /$ $\mathrm{sec}$, maintained for $30 \mathrm{sec}$. This evoked an increase in firing that declined with a time constant of $4.0 \pm$ $0.1 \mathrm{sec}$ (11 units), but the firing rate remained elevated compared to control spontaneous firing. $e$, Effect of rate of stretch on firing. Preparations $(n=7)$ were stretched by $3 \mathrm{~mm}$ at $10 \mu \mathrm{m} / \mathrm{sec}$ ( filled circles), $50 \mu \mathrm{m} / \mathrm{sec}$ (open squares), $100 \mu \mathrm{m} / \mathrm{sec}$ (filled triangles), or $200 \mu \mathrm{m} / \mathrm{sec}$ (open diamonds), and firing frequency was calculated at each length. Consistently larger responses were evoked by the faster rates of stretch for any given length, demonstrating ongoing adaptation during the slower stretches.

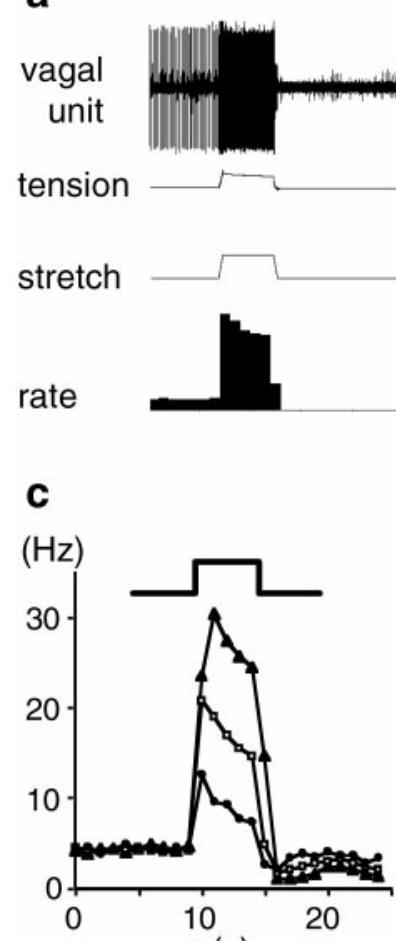

(s) b

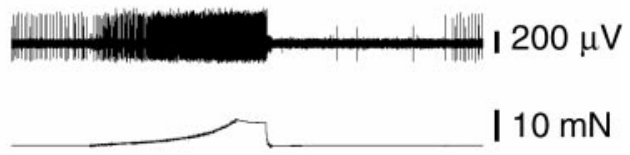

$12 \mathrm{~mm}$ $20 \mathrm{~Hz}$ hand-held, calibrated von Frey hair was applied to the serosal surface of the preparation to identify receptive fields of vagal mechanosensitive afferent neurons. Von Frey hair-sensitive spots (hot spots) and few nearby landmarks were then marked by applying Indian ink on the tip of the hair to the serosal surface. A video camera, positioned above the preparation, was used to capture images of the whole preparation, including ink marks, and to record each site of probing with the von Frey hair.

Anterograde transport and histochemistry. At the end of the recording period, paraffin was removed from the recording chamber and replaced with a $1 \%$ solution of biotinamide (Molecular Probes, Eugene, OR) in an artificial intracellular medium [in mM: 150 monopotassium L-glutamic acid, $7 \mathrm{MgCl}_{2}, 5$ glucose, 1 EGTA, 20 HEPES, and 5 disodium adenosinetriphosphate, $0.02 \%$ saponin, and $1 \%$ dimethylsulfoxide (Tassicker et al., 1999)]. The preparation was covered in supplemented culture medium [DME/F-12 with $10 \%$ fetal bovine serum, $1.8 \mathrm{mM} \mathrm{CaCl}_{2}, 100 \mathrm{IU} / \mathrm{ml}$ penicillin, $100 \mu \mathrm{g} / \mathrm{ml}$ streptomycin, $2.5 \mu \mathrm{g} / \mathrm{ml}$ amphotericin B, $20 \mu \mathrm{g} / \mathrm{ml}$ gentamycin (Cytosystems, New South Wales, Australia), $\mathrm{pH}$ 7.4] and placed on a rocking tray in a humidified incubator with $5 \% \mathrm{CO}_{2}$ in air at $37^{\circ} \mathrm{C}$. After $12-15 \mathrm{hr}$, preparations were fixed in modified Zambonis fixative $(15 \%$ saturated picric acid and $2 \%$ formaldehyde in a $0.1 \mathrm{M}$ phosphate buffer, $\mathrm{pH}$ 7.0), cleared in DMSO for $30 \mathrm{~min}$, and rinsed in PBS. Labeled nerve fibers were visualized with streptavidin-FITC (catalog \#RPN1232; Amersham Life Sciences, Sydney, New South Wales, Australia; 1:50, $4 \mathrm{hr}$ ) or streptavidin-Cy3 (Jackson ImmunoResearch, West Grove, PA; 1:150, $4 \mathrm{hr}$ ). Some preparations were labeled overnight at room temperature with antisera to tyrosine hydroxylase (TH) (catalog \#108460; Incstar, Stillwater, MN; mouse, 1:600), calcitonin gene-related peptide (CGRP) (catalog \#IHC6006; Peninsula Laboratories, Belmont, CA; rabbit, 1:1600), choline acetyltransferase (ChAT) (from Dr. M. Schemann, Hannover Germany, Yeboah; 1:1000), or nitric oxide synthase (NOS) (from Dr. P. Emson, University of Cambridge, Cambridge, UK; K205, $1: 1000)$. These were then rinsed three times in PBS then incubated in appropriate secondary antisera for $2-4 \mathrm{hr}$ (all raised in donkeys and obtained from Jackson ImmunoResearch: anti-sheep IgG-AMCA, 42308: 1:50, anti-rabbit IgG-CY5, 25325, 1:50, anti-mouse IgG-AMCA 19973, 1:200, anti-mouse-FITC 34010 1:100). Preparations were then rinsed again in PBS and mounted in bicarbonate-buffered glycerol, $\mathrm{pH}$ 8.6.

Labeled nerve fibers were analyzed on an Olympus AX70 epifluorescence microscope, using a computerized plotting system which recorded the microscope stage $\mathrm{X}$ and $\mathrm{Y}$ position on two linear scales with $1 \mu \mathrm{m}$ resolution (Mitutoyo, Tokyo, Japan). The coordinates of labeled fibers, IGLEs, viscerof ugal nerve cell bodies, Indian ink marks, and the outlines of the preparation were recorded and reconstructed (Brookes et al., 1992) using commercial graph plotting software (SigmaPlot; Jandel, Corte Madera, CA). Images of labeled neural structures were digitally captured via a Sony (Tokyo, Japan; SSC-M370CE) black and white video camera and recorded on an Apple Power Macintosh 7100 computer using NIH Image version 1.62 software (National Institutes of Health, Bethesda, MD) via a Scion LG-3 frame grabber board (Scion, Frederick, MD). These were then used to create montages to which the computerized reconstructions were fitted, using Canvas 3.05 software (Deneba). Confocal images were obtained on a Bio-Rad (Herts, UK) MRC-1024 combined with an Olympus AX70 microscope equipped with a krypton-argon laser. Optical sections were acquired using a $20 \times$ Olympus oil immersion lens (numerical aperture, 0.8 ) with the confocal iris set to $1-2 \mathrm{~mm}$. The preparations were scanned at $1 \mu \mathrm{m}$ steps to obtain a $\mathrm{Z}$ series of up to 45 optical sections. The microscope was linked to a Compaq Pentium personal computer, running Laser Sharp software (Bio-Rad) for instrument control and data acquisition. Images were combined, cropped, scaled, adjusted for brightness and contrast in NIH Image version 1.62, then scaled, aligned, and labeled in Microsoft Powerpoint 4 and printed on an Epson Stylus Photo EX color printer.

Results are expressed throughout as means \pm SEM, with $n$ referring to the number of animals. Statistical analysis was performed by means of Student's $t$ test for paired and unpaired data. Differences were considered significant if $p<0.05$.

\section{RESULTS}

\section{In vitro recordings from vagal afferent fibers to the guinea pig esophagus}

Preparations had a resting circumference of 5.9-7.2 mm under a resting tension of $1 \mathrm{mN}$. Extracellular recordings were made from fine vagal nerve trunks, $2-4 \mathrm{~mm}$ from the edge of the preparation, and confined to discriminated single axons or "units" whose firing rate was modified by circumferential stretch ( 36 of 41 units, $n=$ 30). Of these stretch-sensitive units, 33 of 36 were spontaneously active, firing at $3.2 \pm 0.5 \mathrm{~Hz}(n=30)$ when the preparation was under minimal resting tension (Fig. $1 a, b$ ).

Rapid circumferential stretch $(5 \mathrm{~mm} / \mathrm{sec})$ evoked an abrupt increase in firing (Fig. 1a) which adapted, with a time constant of $4.0 \pm 0.7 \mathrm{sec}(11$ units, $n=10)$ to a rate higher than resting (Fig. $1 d)$. The circumferential tension of the preparation declined after the initial rise, but with a significantly slower time constant (6.7 \pm $0.6 \mathrm{sec}, n=10, p<0.05$ ), suggesting that neural adaptation may have been partially, but not entirely, attributable to changes in intramural tension. Both phasic and tonic changes in firing were graded with distensions of $1-3 \mathrm{~mm}$ (Fig. 1c), evoking maximum firing rates up to $75 \pm 23 \mathrm{~Hz}$ ( 15 units, $n=12$ ). After returning to resting length, spontaneous firing of units was typically diminished or abolished, returning to control rates over a period of $0.5-30 \mathrm{sec}$ (Fig. $1 a-c$ ). Stretches $>3 \mathrm{~mm}$ sometimes caused irreversible 


$$
\text { a }
$$

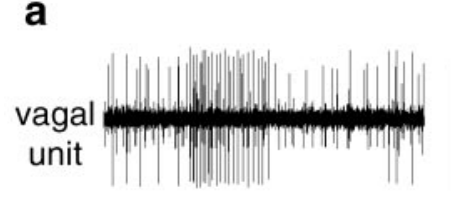

b
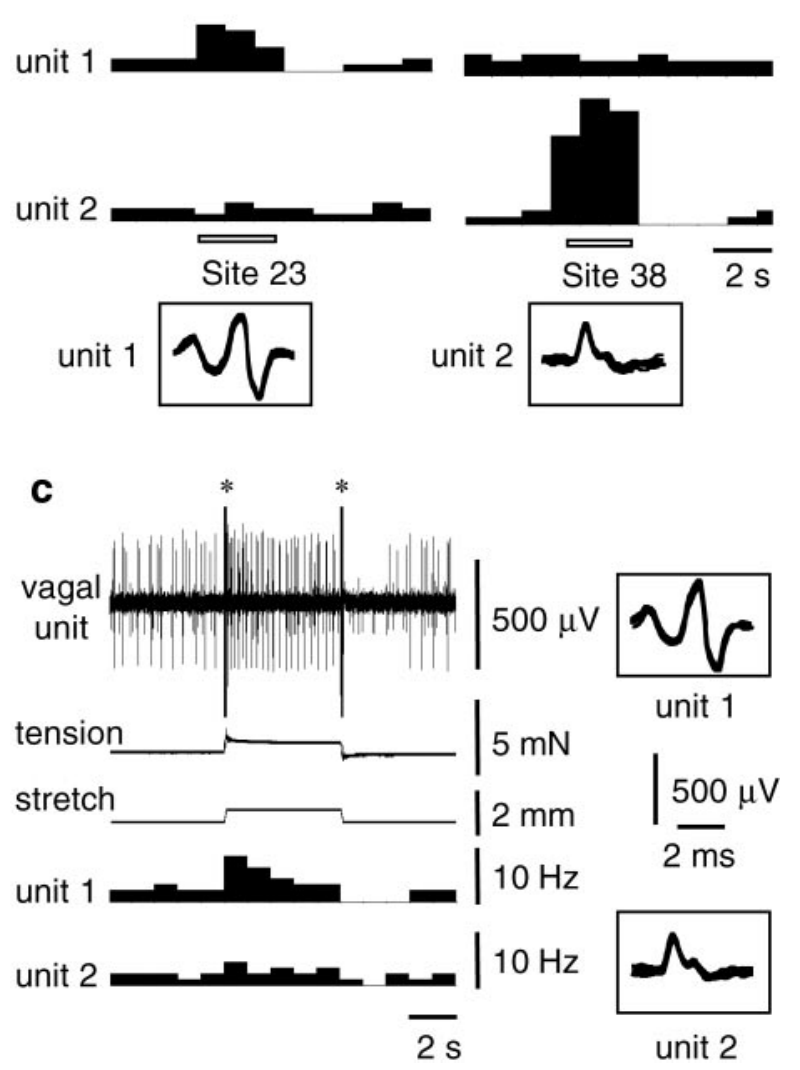

Figure 2. Activation of mechanoreceptors by focal pressure with a calibrated von Frey hair and circumferential stretch. $a, b$, Applying a $0.12 \mathrm{mN}$ calibrated von Frey hair to two sites (designated sites 23 and 38, from the same preparation as Fig. 3) separately activated two units which could be readily discriminated by amplitude. Insets show eight superimposed action potentials activated by von Frey hair. $c$, The same units both responded when the preparation was stretched by a just-suprathreshold length $(0.5$ $\mathrm{mm})$. Calibration bars in $c$ also apply to $a$ and $b$. Insets show eight superimposed successive action potentials from the same units in $a$ and $b$, confirming that the same units were activated by the von Frey hair and by stretch. Insets have separate calibration bars (right side of $c$ ). Asterisks show stepper motor artifacts.

changes in responses and were not used. Stretch at slower rates (10-200 $\mu \mathrm{m} / \mathrm{sec})$ evoked gradually increasing rates of firing. The rate of firing for any particular length was greater when the faster rates of stretch were used $(n=7)$, indicating ongoing adaptation during slow distension (Fig. 1e).

\section{Focal mechanical stimulation}

Serosal probing with a calibrated von Frey hair (which exerted a force of $0.12 \mathrm{mN}$ ) was applied to $100-200$ sites in each preparation and always evoked bursts of firing in just a few sites, which we called hot spots (Figs. $2 a, b, 3 a, b)$. Of a total of 23 units tested, 20 had at least one hot spot $(n=11)$. Probing evoked discharges with maximum firing rates of $58 \pm 14 \mathrm{~Hz}(12$ units, $n=10)$. During maintained probing, there was adaptation of firing but never back to the spontaneous discharge rate. After withdrawal of the probe, spontaneous firing was inhibited or abolished, recovering over a period of $0.5-5 \mathrm{sec}$. In all cases, units that had one or more hot spots were also activated by circumferential stretch of the preparation (Fig. 2c). In 3 of 23 cases, distension-sensitive units were recorded for which no hot spot was located. Given their small size, relative to the preparation, it is possible that some hot spots were missed during these studies.

Hot spots and a few nearby landmarks were marked by applying Indian ink, evaporated onto the tip of the von Frey hair, to the serosal surface of the preparation (Fig. 3a). Multiple sites surrounding each marked hot spot were always probed, but consistently failed to evoke discharge. From this observation, it became clear that the axons that led to the hot spot were not sensitive to the local mechanical distortion evoked by the von Frey hair. From the distance of nonresponding sites to the hot spot it was calculated that the receptive fields were very small, with a maximum diameter ranging from 90 to $330 \mu \mathrm{m}$ (mean, $178 \pm 16 \mu \mathrm{m} ; 17$ units; $n=5$ ). In several units ( 4 of $12 ; n=5$ ) two or more hot spots were found for the same unit; these were spaced up to $2.3 \mathrm{~mm}$ apart (Fig. $3 a, b)$.

The refractory period after probing with a von Frey hair was used to study how multiple transduction sites may contribute to the overall firing of the unit. After recording responses to small rapid circumferential stretches $(0.5-1.5 \mathrm{~mm})$, the von Frey hair was applied to a hot spot, held, and then released. During the ensuing refractory period, the small rapid circumferential stretch was repeated (Fig. 3c). In the first $2 \mathrm{sec}$ of control stretches, firing increased from the spontaneous rate of $3.6 \pm 0.6$ to $7.3 \pm 1.0 \mathrm{~Hz}$, but when preceded by Von Frey hair probing, the same stretch evoked a firing rate of $2.9 \pm 1.3 \mathrm{~Hz}$, which was slightly lower than the spontaneous rate. Thus, increases in firing evoked by distension were significantly reduced after probing with a von Frey hair on a single receptive field ( $p<0.05 ; 11$ units; $n=6$ ). As a control, we examined stretch-evoked responses of other afferent units, which were not affected by applying the von Frey hair. Their responses to stretch were not changed (13 units; $n=6$ ), indicating that the effects of the von Frey hair were specific for the particular unit. Similar inhibition of stretch responses was seen when a von Frey hair was applied to a single site of a unit with two or more identified hot spots. This indicated that hot spots do not generate spikes independently of one another, because desensitization of a single hot spot inhibited the stretch-evoked discharge of the entire unit, including other nonactivated hot spots.

This was investigated further by studying interspike intervals during adapted discharge evoked by $2 \mathrm{~mm}$ distensions maintained for 30-60 sec. For each unit, adapted firing occurred at a very constant frequency $(6.7 \pm 1.5 \mathrm{~Hz} ; 14$ units; $n=9)$ with interspike intervals distributed over a very narrow range (Fig. 4a,b). The mean firing frequency was considerably less than the peak frequency that the unit could generate, indicating spike intervals were not determined by the absolute refractory period of the axon. The absence of closely spaced spikes made it unlikely that hot spots generated spikes independently. This was readily tested by simple modeling. Each simulated spike was set to follow its predecessor by a set interval, with normally distributed temporal "noise" added. The mean interval and variance of the intervals matched those of a real unit (Fig. 4c). Next two spike trains were generated, each with double the mean interspike interval, but the same variance, with the first spikes $180^{\circ}$ out of phase. This simulated two identical, independent spike generating sites, contributing equally to the spike train. Very quickly, spikes from the two generating sites began to move in and out of phase (Fig. $4 d$ ). The resulting interspike intervals had a uniform interval ranging from zero to twice the mean interval (Fig. $4 c$ ). This confirms that it is very unlikely that separate hot spots generate their spikes independently from one another during circumferential stretch.

\section{Anterograde labeling of vagal nerve fibers}

To identify the potential sources of stretch-induced activity recorded from vagal nerve trunks, rapid anterograde labeling was used (Tassicker et al., 1999), in combination with immunohistochemistry for CGRP, TH, NOS, or ChAT. The most numerous labeled afferent nerve endings were those of IGLEs, characterized by their leaf-like ramifying endings on the surfaces of the ganglia, which were seen in 32 of 40 preparations (Figs. $5 a, 6 b, c$ ). In most cases they were restricted to one end of a ganglion and occupied a 
a

Figure 3. Units responded to probing with a calibrated von Frey hair $(0.12$ $\mathrm{mN}$ ) and circumferential stretch. $a$, Video image of preparation with recording chamber to the right and array of hooks connected to the tissue stretcher on the left. Sites that evoked a response (hot spots) are shown as large white circles with black outline, sites that evoked no response (small black dots, white outline), and Indian ink marks (white triangles). Four sites evoked responses in two units: sites 1 , 4 , and 23 all evoked activity in the same unit; site 38 belonged to a second unit (see Fig. 2). Scale bar, $1 \mathrm{~mm}$. $b$, Activity evoked by probing with a von Frey hair at sites 1, 4, and 23, all of which evoked activity in the same unit (insets show 10 superimposed action potentials of the discriminated unit; they are identical). $c$, Control response of another preparation to a small, suprathreshold stretch $(0.5 \mathrm{~mm}$ at $5 \mathrm{~mm} /$ sec) (left panel). Probing with the von Frey hair, at a hot spot, evoked a strong burst of firing in the largeamplitude unit, after which the response to the same stretch was inhibited (right panel). Insets show 10 superimposed action potentials activated by stretch (left inset) and by the von Frey hair (right inset), confirming that the same unit was activated by different stimuli. b
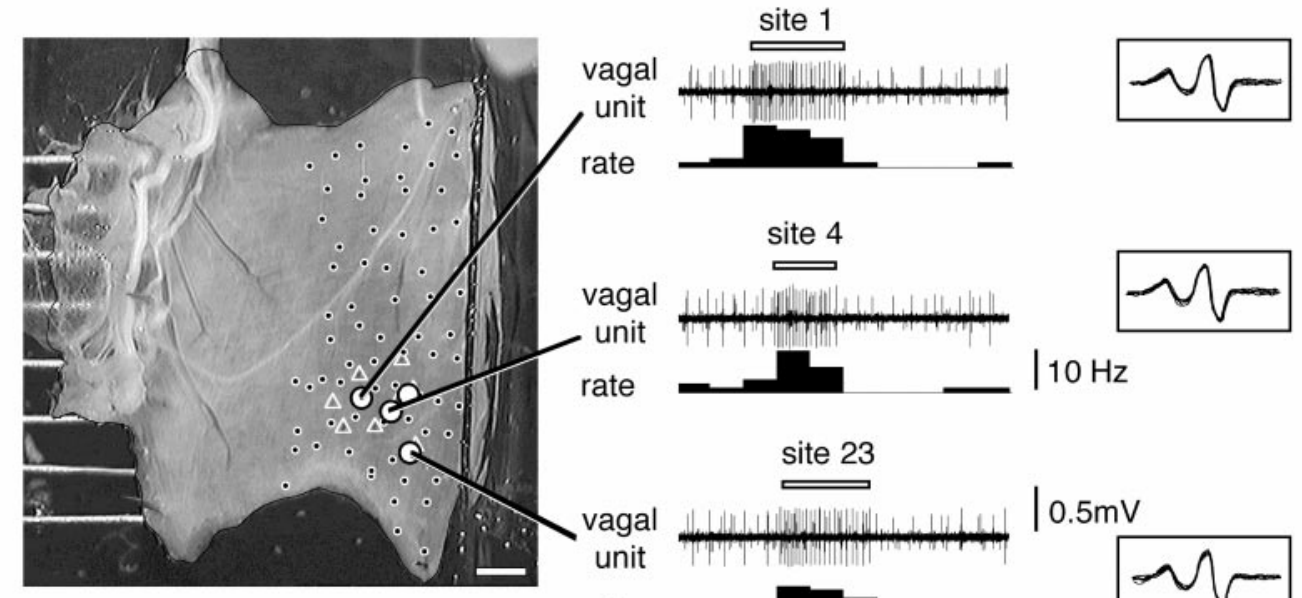

rate
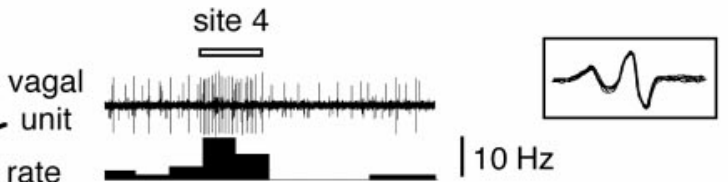

$10 \mathrm{~Hz}$

C
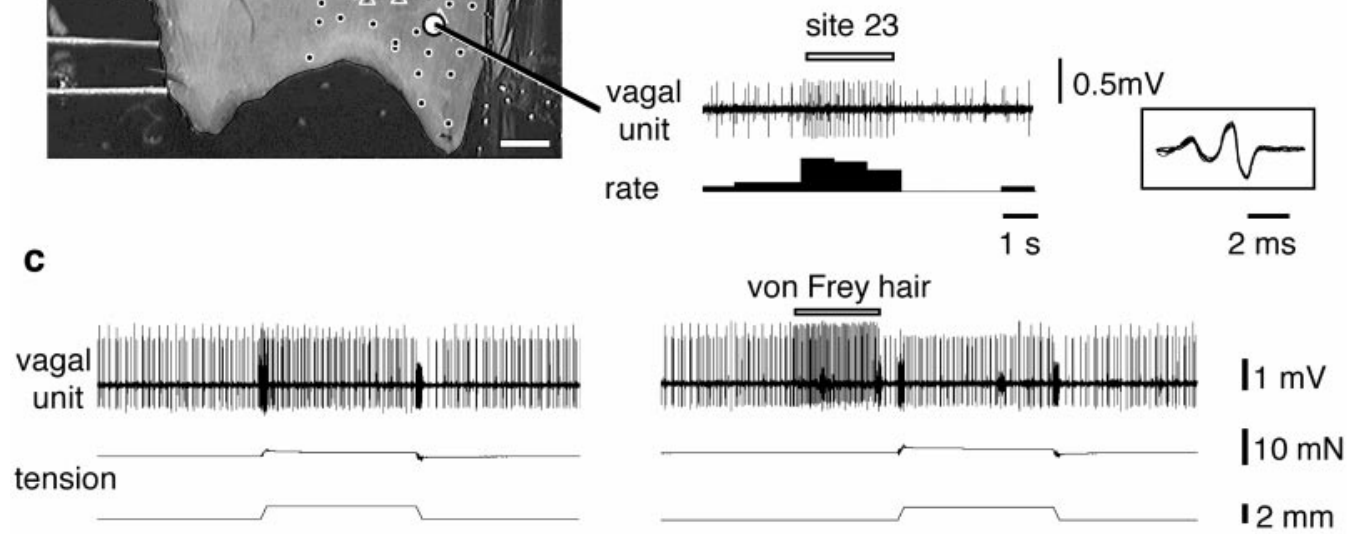

stretch
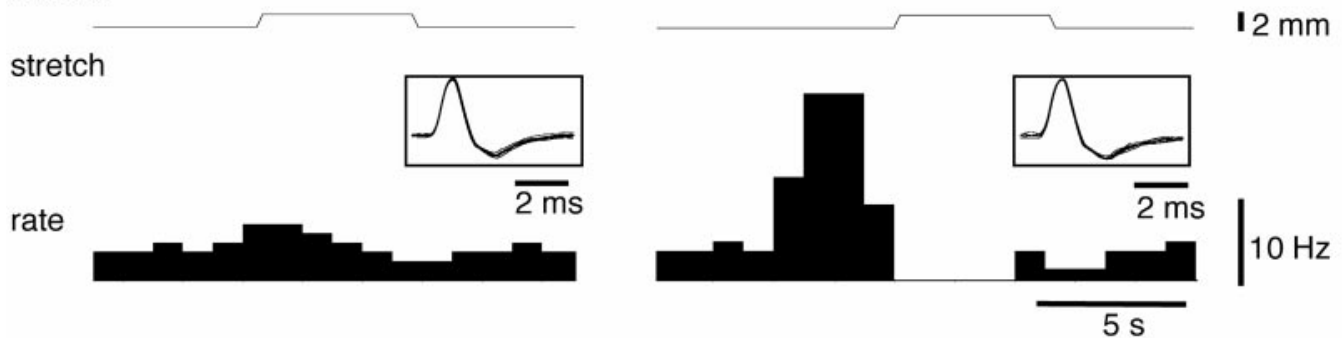

mean area of $6924 \pm 577 \mu \mathrm{m}^{2}$ with a long axis of $147 \pm 8 \mu \mathrm{m}(79$ IGLEs; $n=20$ ). In many cases a single axon gave rise to several IGLEs of various sizes in different ganglia. Bare axons, running for several millimeters parallel to striated muscle fibers of the inner and outer muscle layers were seen in 12 of 22 preparations (Fig. $5 b$ ). These typically branched rather little and never gave rise to motor endplates or IGLEs, as far as we could determine. A total of 93 nerve cell bodies were retrogradely labeled from vagal nerve branches in 17 of 40 preparations (Fig. $5 a$ ). These were small to medium cells (maximal cross-sectional area $651 \pm 35 \mu \mathrm{m}^{2} ; 56$ neurons; $n=12$ ), with a few short lamellar or lobular dendrites and, as far as could be determined, a single axon. Of these cell bodies, 53 of 59 were NOS-immunoreactive, and 5 of 10 also showed ChAT immunoreactivity. These could be the cell bodies of inhibitory motor neurons to the trachea (Fischer et al., 1998; Moffatt et al., 1998) or may project to sympathetic ganglia, mediating reflexes analogous to those described in the intestine (Kreulen and Szurszewski, 1979). Motor endplates were present in 18 of 33 preparations (Fig. 5c). TH- or CGRP-immunoreactive paravascular axons were scarce (4 of 21 preparations). Varicose fibers of vagal preganglionic neurons were also frequently labeled. They branched extensively within myenteric ganglia (33 of 40 preparations; Fig. $5 d$ ), sometimes forming dense baskets of endings around unlabeled nerve cell bodies.

\section{Identification of mechanical transduction sites}

After physiological characterization of mechanoreceptor activity and marking of hot spots with Indian ink, recorded vagal nerve trunks were anterogradely filled with $1 \%$ biotinamide. The average number of visible axons filled from recorded vagal nerve trunks varied from 3 to 15 (mean, $8.7 \pm 1.2 ; n=10$ ). In preliminary experiments at low magnification, each marked hot spot lay close to an anterogradely filled IGLE (mean distance, $108 \pm 48 \mu \mathrm{m} ; n=6$ ). When mapped at higher magnification (Fig. $6 a$ ), the mean distance from hot spots to the nearest IGLE was $73 \pm 14 \mu \mathrm{m}$ (24 hot spots; from 12 units; $n=5$ ). In contrast, the mean distance from randomly generated sites (within the filled area) to the nearest IGLE was significantly greater (374 $\pm 17 \mu \mathrm{m} ; 240$ sites; $n=5 ; p<0.001)$. In several cases, the only nearby filled structure was an IGLE in a myenteric ganglion. It is likely that the distance from IGLEs to hot spots may have been slightly overestimated because Indian Ink marks shifted slightly during tissue handling because of the mobility of the serosa. No other biotinamide-labeled neural structures were associated with hot spots, including bare axons in muscle layers, motor endplates, or postganglionic efferent varicose endings. Four units had two or more hot spots, separated by distances of up to $2.4 \mathrm{~mm}$ (mean, $749 \pm 247 \mu \mathrm{m}$; eight distances; $n=3$ ). In each case an IGLE was associated with each hot spot (Fig. $6 a-c$ ), spaced up to $2.3 \mathrm{~mm}$ apart (mean, $789 \pm 275 \mu \mathrm{m}$; eight pairs of IGLEs; $n=3$ ). In 3 of 11 guinea pigs, nerve cell bodies were filled from recorded nerve trunks, however they were located $697 \pm 234$ $\mu \mathrm{m}$ (five neurons, $n=3$ ) from the nearest recorded hot spot.

\section{DISCUSSION}

Since Pacinian corpuscles were first identified as rapidly adapting mechanoreceptors (Hunt, 1961), the morphology and physiology of many classes of nerve endings in the skin have been identified, providing a firm foundation for the analysis of cutaneous sensation (Iggo, 1985). However, this has not been achieved for visceral afferents, largely because the high density of intrinsic innervation made it impossible to identify unambiguously which nerve ending 

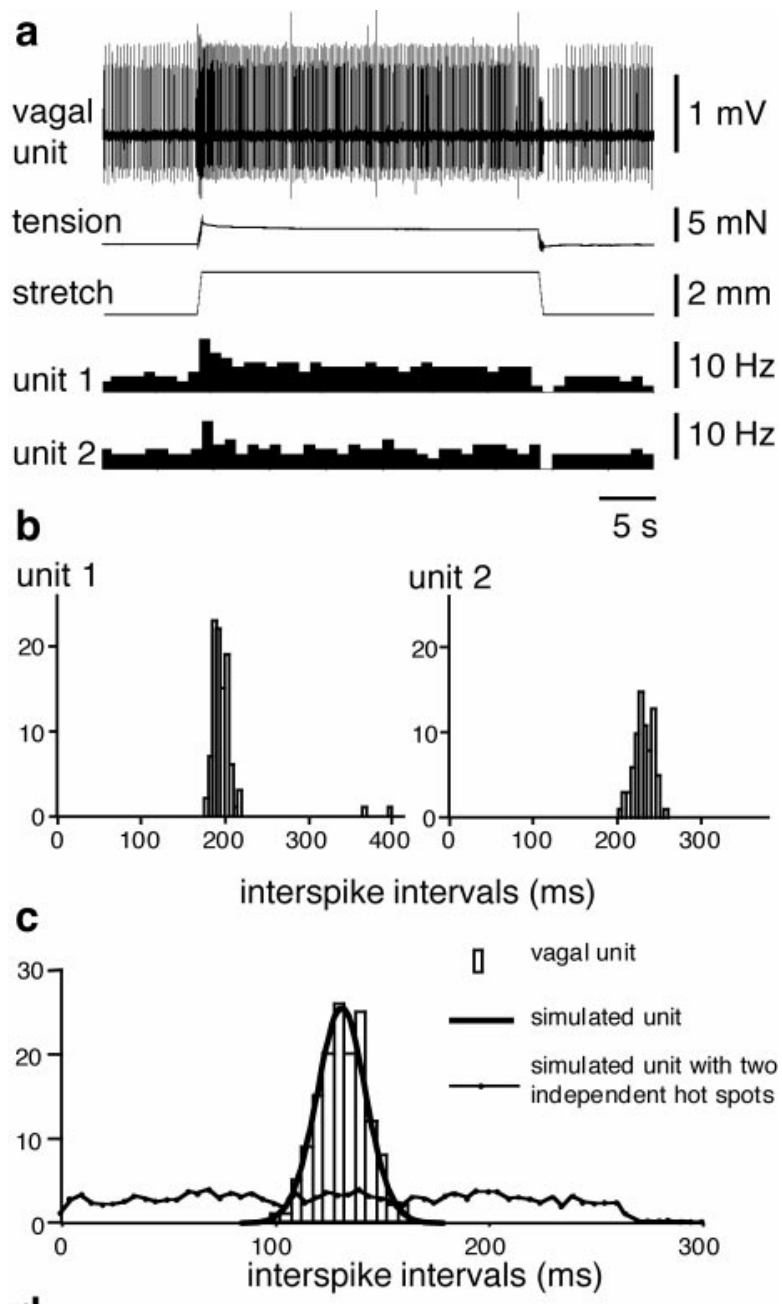

d

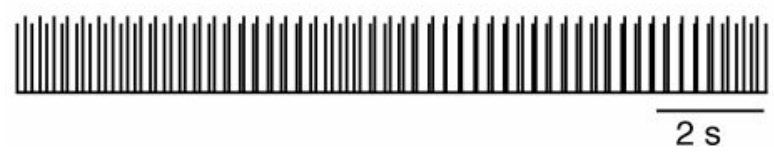

Figure 4. Analysis of interspike intervals during adapted discharge to maintained stretch. $a$, Rapid circumferential stretch $(2 \mathrm{~mm}$ at $5 \mathrm{~mm} / \mathrm{sec}$ for $30 \mathrm{sec}$ ) evoked firing in two units, which adapted to a constant rate after the first $10 \mathrm{sec} . b$ shows the distribution of interspike intervals in the same units from 10 to $30 \mathrm{sec}$ after the onset of stretch. Note the normal distribution and relatively small variance in intervals. $c$, Interspike intervals during adapted discharge in a unit from another preparation, identified as having two hot spots (open bars). A simulated unit, with identical mean and variance is superimposed (thick line). The simulated interspike intervals for a unit with two independent spike-generating sites, with the same variance, is shown as small filled circles; the uniform distribution suggests that multiple hot spots are unlikely to generate action potentials independently, when the preparation is stretched. $d$, Simulated firing of a single unit with two independent spike-generating sites with identical means and variance, initially set $180^{\circ}$ out of phase. Note that spikes from the two sites (visually distinguished by amplitude) rapidly go in and out of phase to generate the uniform distribution of intervals shown in $c$.

had been stimulated. Anterograde filling of small numbers of axons in recorded nerve trunks avoids this problem and has made it possible for us to identify the function of morphologically characterized visceral afferent endings for the first time.

The vagal mechanoreceptors studied here have been recorded electrophysiologically over many years (Iggo, 1955; Leek, 1977; Sengupta and Gebhart, 1994; Page and Blackshaw, 1998), and it is firmly established that they act primarily as in-series tension receptors (Iggo, 1955). Besides their role in mediating sensations such as fullness, when the stomach is distended, extrinsic visceral afferent nerve endings play important roles in regulating the physiological function of the gut (Cervero, 1994; Sengupta and Gebhart, 1994). From the results of the present study, intraganglionic laminar endings are likely to be the first element in physiologically important vagal reflex pathways activated by distension. These include the activation of antral contractions by stomach contents (Andrews et al., 1980), gastric accommodation (Takahashi and Owyang, 1997), duodeno-gastric inhibition (Holzer and Raybould, 1992), and the triggering of transient lower esophageal sphincter relaxations responsible for gastroesophageal reflux (Mittal et al., 1995). The esophageal vagal mechanoreceptors, which were the particular subject of this study, have been shown to modulate the central pattern generator controlling swallowing (Falempin et al., 1986). The recent demonstration that vagal mechanoreceptors can be pharmacologically modulated by $\mathrm{GABA}_{\mathrm{B}}$ receptor agonists (Page and Blackshaw, 1999) raises the possibility of intervening in reflex control of gastrointestinal function at the level of the specialized afferent neurons.

Morphologically, IGLEs were first described in the esophagus (Lawrentjew, 1929), and are known to be present in the smooth muscle esophagus of cat and rhesus monkey (Rodrigo et al., 1975) and in the striated muscle esophagus of the dog (Nonidez, 1946), mouse (Sang and Young, 1998), and guinea pig (present study). In fact, IGLEs are also abundant in the stomach and the small and large intestines (Berthoud et al., 1997), indicating that they play an important role throughout the gastrointestinal tract. Their location, exclusively in myenteric ganglia, led to suggestions that they may be the efferent collaterals of extrinsic afferents (Berthoud and Powley, 1992) or that they may function as mixed afferent and efferent endings (Neuhuber, 1987). Whereas the present study showed that IGLEs are the transduction sites of tension-sensitive mechanoreceptors, it does not exclude the possibility that they may also play an efferent role, because they appear to make close contacts with enteric neurons (Rodrigo et al., 1975; Neuhuber, 1987; Berthoud, 1995). However, a recent study examining fos expression after electrical stimulation of the vagus nerve suggested that such an efferent role for IGLEs or other vagal afferents is likely to be rather limited (Zheng et al., 1997).

Most vagal mechanoreceptors appeared, in our morphological studies, to give rise to several IGLEs in separate ganglia. It is likely that each IGLE is capable of generating action potentials, because von Frey hair probing apparently activated all IGLEs. Several lines of evidence suggested that mechanoreceptor firing evoked by circumferential stretch of the preparation results from coordinated activity between different IGLEs. After maintained pressure applied by a von Frey hair to a single IGLE, spontaneous firing and stretch-evoked responses of the entire unit were inhibited. This suggests that strong activation of one IGLE reduced the excitability of other IGLEs belonging to the same unit. Analysis of interspike intervals during adapted discharge revealed an absence of closely spaced pairs of impulses that would be expected if multiple spikegenerating sites operated independently (Iggo and Muir, 1969). This was supported by statistical modeling, which showed that two independent spike-generating sites do not give rise to the narrow distribution of interspike intervals observed during adapted discharge. One simple explanation for these findings would be that action potentials generated by one IGLE may invade other IGLEs antidromically and reset their discharge. If this is the case, multiple IGLEs may function to "even out" the firing rate for a given stimulus, or, if they have different sensitivities, they may extend the dynamic range over which the unit responds.

This study has identified the peripheral endings of the primary afferent neurons involved in detecting mechanical deformation of the gut wall. These neurons are involved in extrinsic reflex pathways that play important roles in controlling gut function. The approach that we have developed here will allow the systematic identification of extrinsic afferent nerve endings in the gut and other visceral organs. 
Figure 5. Anterograde filling from fine branches of the vagus nerve using biotinamide (see Materials and Methods). a, A retrogradely labeled nerve cell body, several varicose axons, and a faint IGLE are labeled in a myenteric ganglion. $b$, Varicose fibers running within the inner striated muscle layer all arose from a single fiber, which did not give rise to motor endplates. $c$, Three motor endplates arising from a single filled axon are visible within the inner, striated muscle layer. $d$, Extensive varicose branching arising from a single vagal preganglionic efferent fiber ramify through a myenteric ganglion. Such fibers were readily distinguishable from the flattened, leaf-like processes of IGLEs, which were generally confined to the upper and lower surfaces of the ganglia. Scale bars, $50 \mu \mathrm{m}$.
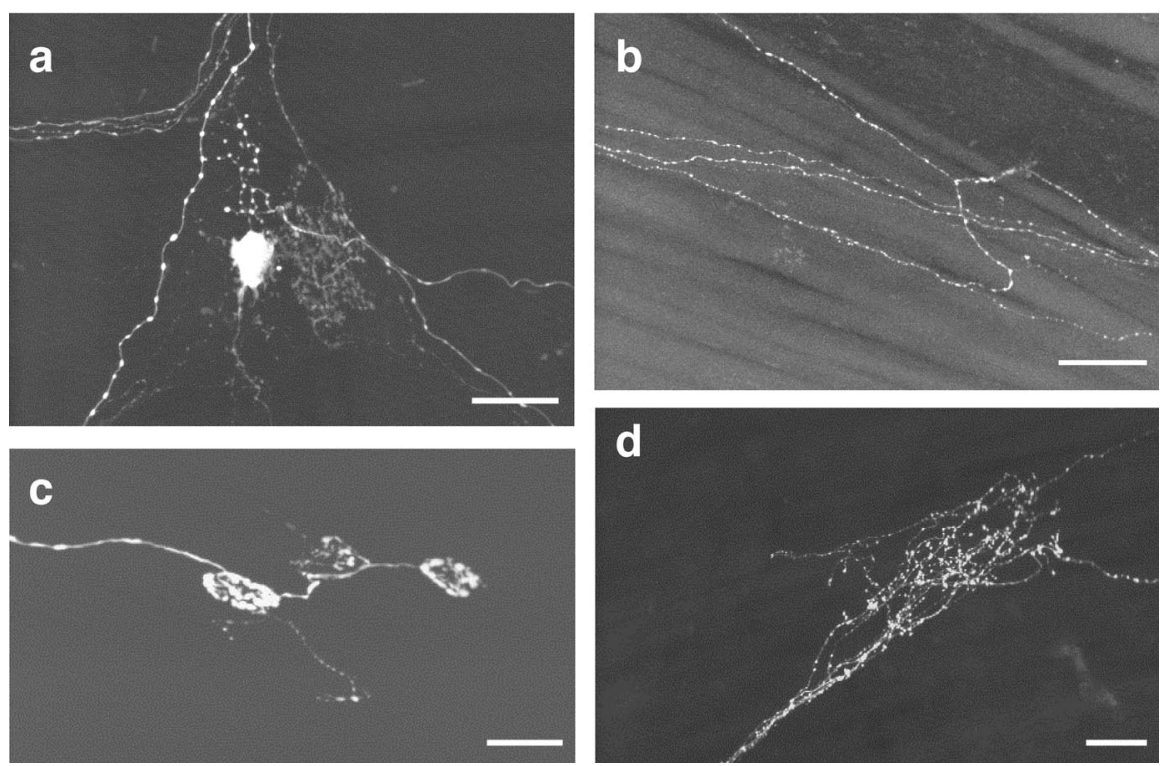

b

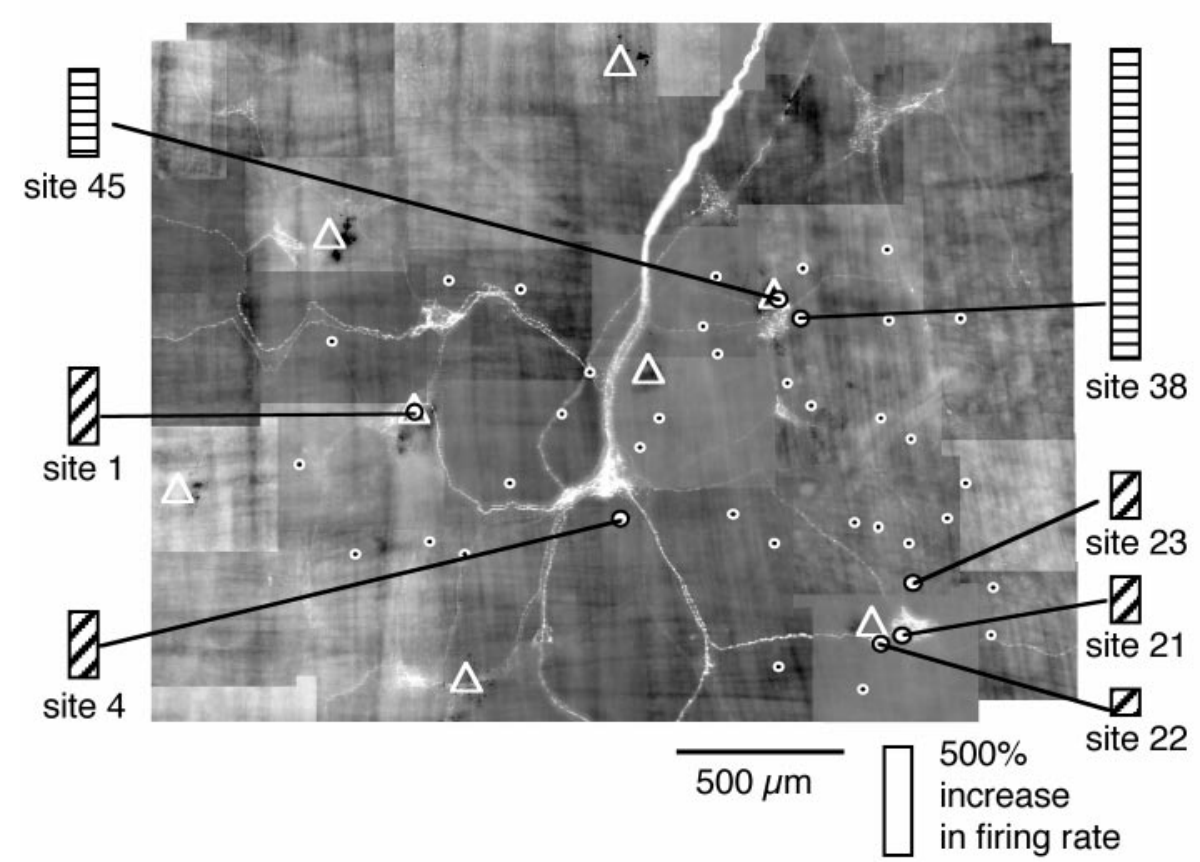

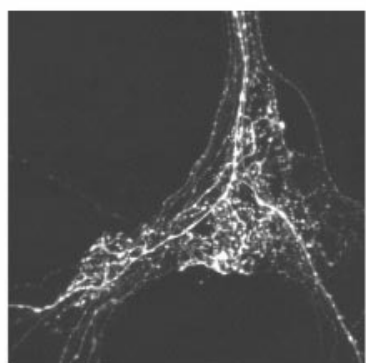

Site $420 \mu \mathrm{m}$

C

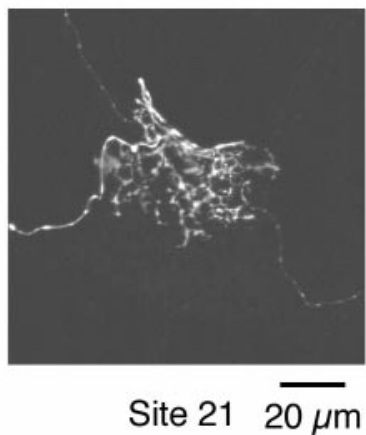

Figure 6. Anterograde filling from a fine vagal nerve trunk from which afferent units had been previously recorded. $a$, Montage of labeled fibers, anterogradely filled from a recorded vagal nerve trunk. Coordinates of hot spots (large white circles with black outline), sites that evoked no response (small black circles, white outline), and Indian ink landmarks (white triangles) were extracted from the video image of the preparation and superimposed on the montage by aligning the Indian ink marks. Note that hot spots are closely related to IGLEs. Details of two sites are shown in $b$ and $c$ that activated the same unit. Images are merged vertical projections of through-ganglion $z$-series taken on a confocal microscope.

\section{REFERENCES}

Andrews PL, Grundy D, Scratcherd T (1980) Reflex excitation of antral motility induced by gastric distension in the ferret. J Physiol (Lond) 298:79-84.

Berthoud HR (1995) Anatomical demonstration of vagal input to nicotinamide acetamide dinucleotide phosphate diaphorase-positive (nitrergic) neurons in rat fundic stomach. J Comp Neurol 358:428-439.

Berthoud HR, Patterson LM (1996) Anatomical relationship between vagal afferent fibers and CCK-immunoreactive entero-endocrine cells in the rat small intestinal mucosa. Acta Anat 156:123-131.

Berthoud HR, Powley TL (1992) Vagal afferent innervation of the rat fundic stomach: morphological characterization of the gastric tension receptor. J Comp Neurol 319:261-276.

Berthoud HR, Patterson LM, Neumann F, Neuhuber WL (1997) Distri- bution and structure of vagal afferent intraganglionic laminar endings (IGLEs) in the rat gastrointestinal tract. Anat Embryol 195:183-191.

Brookes SJH, Song ZM, Steele PA, Costa M (1992) Identification of motor neurons to the longitudinal muscle of the guinea pig ileum. Gastroenterology 103:961-973.

Brookes SJH, Chen BN, Costa M, Humphreys CMS (1999) Initiation of peristalsis by circumferential stretch in flat sheets of guinea-pig ileum. J Physiol (Lond) 516.2:525-538.

Cervero F (1994) Sensory innervation of the viscera-peripheral basis of visceral pain. Physiol Rev 74:95-138.

Clerc N, Condamin M (1987) Selective labeling of vagal sensory nerve fibers in the lower esophageal sphincter with anterogradely transported WGA-HRP. Brain Res 424:216-224.

Falempin M, Madhloum A, Rousseau JP (1986) Effects of vagal deaffer- 
entation on oesophageal motility and transit in the sheep. J Physiol (Lond) 372:425-436.

Fischer A, Canning BJ, Undem BJ, Kummer W (1998) Evidence for an esophageal origin of VIP-IR and NO synthase-IR nerves innervating the guinea pig trachealis: a retrograde neuronal tracing and immunohistochemical analysis. J Comp Neurol 394:326-334.

Furness JB, Costa M (1987) The enteric nervous system. Edinburgh: Churchill-Livingstone.

Holzer HH, Raybould HE (1992) Vagal and splanchnic sensory pathways mediate inhibition of gastric motility induced by duodenal distension. Am J Physiol 25:G603-G608.

Hunt CC (1961) On the nature of vibration receptors in the hind limb of the cat. J Physiol (Lond) 155:175-186.

Iggo A (1955) Tension receptors in the stomach and the urinary bladder. J Physiol (Lond) 128:593-607.

Iggo A (1985) Sensory receptors in the skin of mammals and their sensory functions. Rev Neurol 141:599-613.

Iggo A, Muir AR (1969) The structure and function of a slowly adapting touch corpuscle in hairy skin. J Physiol (Lond) 200:763-796.

Kreulen DL, Szurszewski JH (1979) Reflex pathways in the abdominal prevertebral ganglia: evidence for a colo-colonic inhibitory reflex. J Physiol (Lond) 295:21-32.

Lawrentjew BJ (1929) Experimentell-morphologische Studien über den Aufbau des Ganglien des Spieserohre nebst einigen Bemerkungen über das Vorkommen und die Verteilung zweier Arten von Nervenzellen im autonomen Nervensystem. Z Zellforsch Mikrosk Anat Forsch 18:233-262.

Leek BF (1977) Abdominal and pelvic visceral receptors. Br Med Bull 33:163-168

Mittal RK, Holloway RH, Penagini R, Blackshaw LA, Dent J (1995) Transient lower esophageal sphincter relaxation. Gastroenterology 109:601-610.

Moffatt JD, Dumsday B, McLean JR (1998) Non-adrenergic, noncholinergic neurons innervating the guinea-pig trachea are located in the oesophagus: evidence from retrograde neuronal tracing. Neurosci Lett 248:37-40.
Neuhuber WL (1987) Sensory vagal innervation of the rat esophagus and cardia: a light and electron microscope anterograde tracing study. J Autonom Nerv Syst 20:243-255.

Nonidez JF (1946) Afferent nerves in the intermuscular plexus of the dog's oesophagus. J Comp Neurol 85:177-189.

Page AJ, Blackshaw LA (1998) An in vitro study of the properties of vagal afferent fibres innervating the ferret oesophagus and stomach. J Physiol (Lond) 512:907-916.

Page AJ, Blackshaw LA (1999) GABA(B) receptors inhibit mechanosensitivity of primary afferent endings. J Neurosci 19:8597-8602.

Phillips RJ, Baronowsky EA, Powley TL (1997) Afferent innervation of gastrointestinal tract smooth muscle by the hepatic branch of the vagus. J Comp Neurol 384:248-270.

Rodrigo J, Hernandez CJ, Vidal MA, Pedrosa JA (1975) Vegetative innervation of the esophagus. II. Intraganglionic laminar endings. Acta Anat 92:79-100.

Sang Q, Young HM (1998) The origin and development of the vagal and spinal innervation of the external muscle of the mouse esophagus. Brain Res 809:253-268.

Sengupta JN, Gebhart G (1994) Gastrointestinal afferent fibers and sensation. In: Physiology of the gastrointestinal tract. Vol 1 (Johnson LR, ed), pp 483-520. New York: Raven.

Takahashi T, Owyang C (1997) Characterization of vagal pathways mediating gastric accommodation reflex in rats. J Physiol (Lond) 504:479-488.

Talley NJ, Gabriel SE, Harmsen WS, Zinsmeister AR, Evans RW (1995) Medical costs in community subjects with irritable bowel syndrome. Gastroenterology 109:1736-1741.

Tassicker BC, Hennig GW, Costa M, Brookes SJH (1999) Rapid anterograde and retrograde tracing from mesenteric nerve trunks to the guinea pig small intestine in vitro. Cell Tissue Res 295:437-452.

Zheng HY, Lauve A, Patterson LM, Berthoud HR (1997) Limited excitatory local effector function of gastric vagal afferent intraganglionic terminals in rats. Am J Physiol Gastrointest Liver Physiol 36:G661G669. 